\title{
Projections of Gibbs Measures May Be Non-Gibbsian`
}

\author{
Roberto H. Schonmann \\ Department of Mathematics, Cornell University, Ithaca, NY 14853, USA and IME-USP, Caixa Postal \\ 20570, 01000 São Paulo SP, Brazil
}

\begin{abstract}
Consider the + phase of the two dimensional nearest neighbor ferromagnetic Ising model at a temperature below $T_{c}$. Let $v_{+}$be the restriction of this measure to a coordinate axis. We prove that there is no one dimensional translation invariant summable interaction for which $v_{+}$is a Gibbs measure. This is proven by showing that if such an interaction existed, $v_{+}$would have large deviation properties different from those it actually has. Percolation methods are used in the proof.
\end{abstract}

In this note we consider the two dimensional nearest neighbor ferromagnetic Ising model. The formal Hamiltonian may be written as

$$
H_{\text {Ising }}(\sigma)=-(J / 2) \sum_{\langle x, y\rangle} \sigma_{x} \sigma_{y},
$$

where $J>0, \sigma=\left(\sigma_{x}\right)_{x \in Z^{2}}, \sigma_{x}=\mp 1$ and the sum runs over the pairs of nearest neighbor sites of $Z^{2}$.

Let $\Lambda_{n}$ be the box $\{-n, \ldots, n\}^{2}$ and $\mu_{n,+, J}$ be the Gibbs measure with + boundary conditions on $\left(\Lambda_{n}\right)^{c}$. (Throughout this paper we will always take the usual factor $\beta$ equal to one in the definition of Gibbs measures). As $n \rightarrow \infty, \mu_{n,+, J}$ converges weakly to the + phase $\mu_{+, J} . \mu_{n,-, J}$ and $\mu_{-, J}$ have analogous definitions. Set

$$
J_{c}=\inf \left\{J>0 ; \mu_{-, J} \neq \mu_{+, J}\right\} .
$$

Given $A \subset Z^{2}$, let $\mathscr{F}(A)$ be the $\sigma$-algebra generated by the random variables $\left\{\sigma_{x}: x \in A\right\}$. Denote by $L$ the line $Z \times\{0\}$ and let $v_{ \pm, J}$ be the restriction of $\mu_{ \pm, J}$ to $\mathscr{F}(L)$, i.e., the measure induced by $\mu_{ \pm, J}$ on $L$.

We will prove below

Theorem. Assume $J>J_{c}$. Then there is no one dimensional translation invariant summable interaction such that $v_{+, J}$ is a Gibbs measure for this interaction.

\footnotetext{
* Work supported by the U.S. Army Research Office through the Mathematical Sciences Institute at Cornell and by a NSF grant to Cornell. This work was finished while the author was visiting Rutgers University, being supported by the NSF grant 86-12369
} 
Our motivation to consider this type of question arises from the desire of better understanding how generally a measure is Gibbsian. (See open problem B1 in [Rue], p. 169). While the result in this paper is only a negative answer in a very particular case, it shows that even measures that appear naturally in connection with simple statistical mechanics models may be non-Gibbsian (in the sense stated in the Theorem). This possibility was pointed out in [GP1, GP2 and Gri] (see also [Isr 3]), in connection with problems related to real space renormalization group procedures. In these papers restrictions of Gibbs measures to sublattices obtained from the original one by "decimation" were for instance discussed. It is possible that the methods used in the present paper may help to elucidate some of the problems raised in those papers. We have also some hope that the methods used here can be adapted to deal with measures which are invariant for some stochastic dynamics, for which it is in general interesting and hard to decide whether the measure is Gibbsian or not (see [Kun and LS]). One should remark that if a larger class of interactions and a more general definition of Gibbs measure is adopted [Isr 1, Isr 2] one can find an interaction for which not just one measure, but all the measures in an arbitrarily large finite set are Gibbsian. These interactions nevertheless give rise to all sorts of pathologies and seem therefore to be of little physical significance (see the remarks on pages 119 and 120 of [Isr 2]). We should also remark that in [Koz and Sul], necessary and sufficient conditions for measures to be in certain classes of Gibbsian measures are provided. But these conditions, which involve continuity of conditional probabilities, are in general hard to verify. We were not able to use them in this paper and in some other cases (see [LS]).

Next we recall the definition of the class of interactions mentioned in the Theorem. For this purpose let $\mathscr{P}_{f}(Z)$ be the set of finite subsets of $Z$ and $\Omega=\{-1,+1\}^{Z}$. Given $V \in \mathscr{P}_{f}(Z)$ and $i \in Z$, set

$$
V+i=\{j \in Z: j-i \in V\} \text {. }
$$

Given now $\omega \in \Omega$, we define $\tau_{i} \omega$ by $\left(\tau_{i} \omega\right)_{j}=\omega_{j-i}$. A one dimensional translation invariant summable interaction is a function $I_{W}(\omega), W \in \mathscr{P}_{f}(Z), \omega \in \Omega$ such that

(i) $I_{W+i}\left(\tau_{i} \omega\right)=I_{W}(\omega)$,

(ii) $\sum_{W: O \in W} \sup _{\omega}\left|I_{W}(\omega)\right|<\infty$.

Remark. (i) and (ii) imply that for any $V \in \mathscr{P}_{f}(Z)$,

$$
\sum_{W: W \cap V \neq \phi} \sup _{\omega}\left|I_{W}(\omega)\right|<\infty .
$$

A measure $\mu$ is a Gibbs measure for the interaction $I_{W}(\omega)$ if for every $V \in \mathscr{P}_{f}(Z)$ and $\sigma \in \Omega$ a version of the conditional probability

$$
\mu\left\{\omega: \omega_{i}=\sigma_{i} \text { for } i \in V \mid \omega_{i}=\sigma_{i} \text { for } i \in V^{c}\right\}
$$

is given by

$$
e^{-H_{V}(\sigma)} / \sum_{\sigma^{\prime} \in B_{V}(\sigma)} e^{-H_{V}\left(\sigma^{\prime}\right)}
$$


where

$$
H_{V}(\sigma)=\sum_{W: W \cap V \neq \phi} I_{W}(\sigma)
$$

and

$$
B_{V}(\sigma)=\left\{\sigma^{\prime} \in \Omega: \sigma_{i}^{\prime}=\sigma_{i} \text { for } i \in Z \backslash V\right\} .
$$

We turn now to the proof of the Theorem. Since $J>J_{c}$ is fixed, it is generally omitted.

Define

$$
S_{n}=\{-n, \ldots, n\}
$$

(below we will redefine $S_{n}$ as a subset of $Z^{2}$, but this should not give rise to any confusion). And $m^{*}=\int \sigma_{0} d \mu_{+}$. Our reasoning is as follows. The following large deviations property for $v_{+}$is known to hold ([Sch, CCS ]):

Proposition 1. Assume $J>J_{c}$, then there exists a convex function $\varphi:[-1,1] \rightarrow$ $[0, \infty)$ (which depends on $J$ ) such that $\varphi(m)=0$ if and only if $m=m^{*}$ and

$$
\lim _{n \rightarrow \infty}\left|S_{n}\right|^{-1} \log v_{+, J}\left\{\omega:\left|S_{n}\right|^{-1} \sum_{i \in S_{n}} \omega_{i} \in(a, b)\right\}=-\inf _{a<m<b} \varphi(m)
$$

for any $-1 \leqq a<b \leqq 1$.

On the other hand, in [FO and O11] large deviation properties were proved for the empirical field associated with a large class of Gibbs measures, including all the Gibbs measures for translation invariant summable interactions, considered above (the same result was also announced independently in [Com]). It follows from this result, via a contraction principle (see Sect. 6 in [O11]) that any Gibbs measure in this class satisfies a large deviation property of the form (2) above, but the corresponding function $\varphi(m)$ has a unique zero if and only if there is no other translation invariant Gibbs measure for the same interaction having a different density (see the last remark in Sect. 6 of [O11]). Therefore Theorem 1 will be proved once we prove

Proposition 2. Assume $J>J_{c}$. If $v_{+, J}$ were a Gibbs measure corresponding to a translation, invariant summable interaction, $I_{W}(\omega)$, then $v_{-, J}$ would also be a Gibbs measure for the same interaction.

Our proof of Proposition 2 is based on a simple idea exploring a result in [Rus], but the technicalities may obscure the arguments. Therefore we first sketch our approach. We want to show that for any $\ell, v_{+}$conditioned on $\left\{\omega_{i}=-1\right.$ if $|i| \geqq \ell\}$ is stochastically lower than $v_{\text {. }}$. It would then follow easily that as $\ell \rightarrow \infty$ this conditional measure converges to $v_{-}$, implying what we want to prove. But the procedure above involves conditioning on a set of $v_{+}$probability zero. So we have to introduce a cut-off, conditioning on $\left\{\omega_{i}=-1\right.$ if $\left.\ell \leqq|i| \leqq n\right\}$ for appropriate $n$ and use carefully the definition of a Gibbs measure. This is done in the proof of Lemma 2 below. We start with several definitions (some appeared before and are recalled) and Lemma 1, where we use results and percolation techniques from [Rus]. 
Subsets of $Z^{2}$ :

$$
\begin{aligned}
\Lambda_{n} & =\{-n, \ldots, n\}^{2}, \\
L & =Z \times\{0\}, \\
S_{\ell} & =\{-\ell, \ldots, \ell\} \times\{0\} \text { for } \ell \geqq 0, \\
R_{\ell, n} & =S_{n} \backslash S_{\ell-1} \text { for } 1 \leqq \ell<n, \\
R_{\ell} & =L \backslash S_{\ell-1} \text { for } \ell \geqq 1, \quad R_{0}=L .
\end{aligned}
$$

Measures:

i) $\mu_{n}^{\ell}$ is the Gibbs measure for the two dimensional Ising model with "boundary condition": $\sigma_{x}=-1$ if $x \in R_{\ell}, \sigma_{x}=+1$ if $x \in\left(\Lambda_{n}\right)^{c} \backslash R_{\ell}$.

ii) By FKG, as $n \rightarrow \infty, \mu_{n}^{\ell}$ converges weakly to a measure $\mu^{\ell}$.

$\sigma$-algebras:

$\mathscr{F}(A)=\sigma$-algebra generated by the spins $\left\{\sigma_{x}: x \in A\right\}$,

$\mathscr{F}_{\infty}=\bigcap_{\substack{A \subset Z^{2} \\ \text { finite }}} \mathscr{F}\left(A^{c}\right)$.

Inequalities:

i) Given two measures $\mu_{1}$ and $\mu_{2}$ on $\mathscr{F}\left(Z^{2}\right)$, we write $\mu_{1} \leqq \mu_{2}$ if $\int f d \mu_{1} \leqq \int f d \mu_{2}$ for every coordinatewise non-decreasing continuous function $f:\{-1,1\}^{\overline{Z^{2}}} \rightarrow \mathbb{R}$.

ii) An event is said to be positive if its indicator function is a coordinativewise non-decreasing function.

Lemma 1. For any $\ell \geqq 0, \mu^{\ell} \leqq \mu_{-}$.

Proof. By FKG, $\mu^{\ell}$ is an extremal Gibbs measure for the system on the lattice $Z^{2} \backslash R_{\ell}$, with formal Hamiltonian

where

$$
H^{\ell}(\sigma)=H_{\text {Ising }}(\sigma)+\sum_{x \in Z^{2}} h_{x} \sigma_{x}
$$

$$
\left.h_{x}=(J / 2) \text { (number of nearest neighbors of } x \text { in } R_{\ell}\right) \text {. }
$$

Indeed, if $\mu$ is any Gibbs measure for this system, $\mu \leqq \mu^{\ell}$, and therefore $\mu^{\ell}$ cannot be decomposed into a non-trivial convex linear combination of these measures. From the extremality, it follows that $\mathscr{F}_{\infty}$ is trivial when measured by $\mu^{\ell}$ ([Rue], Theorem 1.11).

We will use now percolation techniques from [Rus]. The reader should consult this paper for the definitions of terms like "infinite cluster," "*circuit," etc, used below.

Let $C_{u}$ [respectively $C_{\ell}$ ] be the event that there is an infinite $(+)$ cluster in the upper [respectively lower] half plane $Z \times\{1,2, \ldots\}$ [respectively $Z \times\{-1,-2, \ldots\}$ ]. Then

$$
\begin{aligned}
\mu^{\ell}\left(\left(C_{u}\right)^{c}\right) & \geqq \mu^{\ell}\left(\left(C_{u}\right)^{c} \mid\left\{\sigma_{x}=-1 \text { for } x \in S_{\ell-1}\right\}\right) \mu^{\ell}\left\{\sigma_{x}=-1 \text { for } x \in S_{\ell-1}\right\} \\
& =\mu^{0}\left(\left(C_{u}\right)^{c}\right) \cdot \mu^{\ell}\left\{\sigma_{x}^{x}=-1 \text { for } x \in S_{\ell-1}\right\} .
\end{aligned}
$$

But Lemma 8 in [Rus] states that the first factor in the right-hand side above is 
one, while the second one is clearly positive. Hence $\mu^{\ell}\left(\left(C_{u}\right)^{c}\right)>0$. Now the triviality of $\mathscr{F}_{\infty}$ implies $\mu^{\ell}\left(\left(C_{u}\right)^{c}\right)=1$. Analogously $\mu^{\ell}\left(\left(C_{\ell}\right)^{c}\right)=1$.

Now it is easy to finish the proof, with a standard argument. The absence of infinite $(+)$ clusters in the upper and the lower half planes implies the occurrence of $a^{*}$ circuit of - surrounding any given finite region (remember that the spins are constrained to be -1 on $R_{\ell}$ ). This fact implies $\mu^{\ell} \leqq \mu_{-}$(see the proof of Lemma 1 in [Rus] for details).

We will denote by $\mathbf{E}_{+}$expected values with respect to $v_{+}$. Saying that $v_{+}$is a Gibbs measure for the interaction $I_{W}(\omega)$ is clearly equivalent to requiring that for any $V \in \mathscr{P}_{f}(Z)$ and any event $E \in \mathscr{F}(V)$,

$$
f_{V, E}(\sigma)=\frac{\sum_{\sigma^{\prime} \in B_{V}(\sigma) \cap E} e^{-H_{V}\left(\sigma^{\prime}\right)}}{\sum_{\sigma^{\prime \prime} \in B_{V}(\sigma)} e^{-H_{V}\left(\sigma^{\prime \prime}\right)}}
$$

is a version of the conditional expectation

$$
\mathbf{E}_{+}\left(\mathbf{1}_{E} \mid \mathscr{F}\left(V^{c}\right)\right)(\sigma)
$$

where, as before

$$
B_{V}(\sigma)=\left\{\sigma^{\prime} \in \Omega: \sigma_{i}^{\prime}=\sigma_{i} \text { if } i \in Z \backslash V\right\},
$$

and $\mathbf{1}_{E}$ is the indicator function of the event $E$. Below we use the notation $f_{\ell, E}(\sigma)$ for $f_{V, D}(\sigma)$ when $V=S_{\ell}$. We denote by $\sigma_{-}$the configuration identically -1 on $Z$. Recall also that $\mu_{\ell,-}$ is the Gibbs measure for the two dimensional Ising model with-boundary conditions outside $\Lambda_{\ell}=\{-\ell, \ldots, \ell\}^{2}$ and that $\mu_{-}$is the limit of $\mu_{\ell,-}$ as $\ell \rightarrow \infty$.

Lemma 2. Under the assumptions of Proposition 2, if $E \in \mathscr{F}\left(S_{\ell}\right)$ is a positive event, then

$$
\mu_{\ell,-}(E) \leqq f_{\ell, E}\left(\sigma_{-}\right) \leqq \mu_{-}(E),
$$

where $f_{\ell, E}$ corresponds via (3) to the interaction $I_{W}(\omega)$, supposed to exist in the assumptions of Proposition 2.

Proof. Take an $\varepsilon>0$. By the definition of $\mu^{\ell}$, there exists an $n_{0}$ such that if $n \geqq n_{0}$,

$$
\mu_{n}^{\ell}(E) \leqq \mu^{\ell}(E)+\varepsilon / 3 .
$$

(1) implies that $f_{\ell, E}(\cdot)$ is continuous. Therefore there exists $n_{1}$ such that if $\sigma^{\prime}$ satisfies $\sigma_{i}^{\prime}=-1$ for $i \in S_{n_{1}}$, then

$$
\left|f_{\ell, E}\left(\sigma^{\prime}\right)-f_{\ell, E}\left(\sigma_{-}\right)\right| \leqq \varepsilon / 3 .
$$

Set $\bar{n}=\max \left(n_{0}, n_{1}\right)$.

By an application of the theorem of convergence of martingales (see Theorem 35.5 in [Bil]) we have

$$
\mathbf{E}_{+}\left(\mathbf{1}_{E} \mid \mathscr{F}\left(R_{\ell, N}\right)\right)(\sigma) \rightarrow f_{\ell, E}(\sigma)
$$

as $N \rightarrow \infty$, for almost every $\sigma$. But for any $n$ the set $D_{n}=\left\{\sigma^{\prime} \in \Omega: \sigma_{i}^{\prime}=-1\right.$ if $\left.i \in S_{n}\right\}$ has $v_{+}$positive measure. Therefore there exists $\sigma^{\prime} \in D_{n}$ such that (6) holds when 
$\sigma=\sigma^{\prime}$. Take now $N \geqq \bar{n}$ such that

Using (5) and (7)

$$
\left|\mathbf{E}_{+}\left(\mathbf{1}_{E} \mid \mathscr{F}\left(R_{\ell, N}\right)\right)\left(\sigma^{\prime}\right)-f_{\ell, E}\left(\sigma^{\prime}\right)\right| \leqq \varepsilon / 3
$$

By FKG

$$
\begin{aligned}
f_{\ell, E}\left(\sigma_{-}\right) & \leqq f_{\ell, E}\left(\sigma^{\prime}\right)+\varepsilon / 3 \leqq \mathbf{E}_{+}\left(\mathbf{1}_{E} \mid \mathscr{F}\left(R_{\ell, N}\right)\right)\left(\sigma^{\prime}\right)+2 \varepsilon / 3 \\
& =\mu_{+}\left(E \mid\left\{\omega: \omega_{x}=\sigma_{x}^{\prime} \text { for } x \in R_{\ell, N}\right\}\right)+2 \varepsilon / 3 .
\end{aligned}
$$

$$
f_{\ell, E}\left(\sigma_{-}\right) \leqq \mu_{n_{0}}^{\ell}(E)+2 \varepsilon / 3
$$

Using (4) and Lemma 1,

$$
f_{\ell, E}\left(\sigma_{-}\right) \leqq \mu^{\ell}(E)+\varepsilon \leqq \mu_{-}(E)+\varepsilon .
$$

Since $\varepsilon$ is arbitrary, it follows that

$$
f_{\ell, E}\left(\sigma_{-}\right) \leqq \mu_{-}(E) .
$$

The proof of the lower bound is analogous but simpler since, clearly, by FKG, $\mu_{\ell,-} \leqq \mu_{n}^{\ell}$ :

$$
\begin{aligned}
f_{\ell, E}\left(\sigma_{-}\right) & \geqq f_{\ell, E}\left(\sigma^{\prime}\right)-\varepsilon / 3 \geqq \mathbf{E}\left(\mathbf{1}_{E} \mid \mathscr{F}\left(R_{\ell, N}\right)\right)\left(\sigma^{\prime}\right)-2 \varepsilon / 3 \\
& \geqq \mu_{\ell,-}\left(\sigma^{\prime}\right)-2 \varepsilon / 3 .
\end{aligned}
$$

Proof of Proposition 2. From Lemma 2,

$$
\lim _{\ell \rightarrow \infty} f_{\ell, E}\left(\sigma_{-}\right)=\mu_{-}(E)=v_{-}(E),
$$

for any positive event $E$ which depends only on finitely many spins. By standard arguments, using inclusion-exclusion, we can drop the condition of positivity of $E$. But this implies (see Theorem 1.9 in [Rue]) that $v_{-}$is a Gibbs measure with respect to the interaction $I_{W}(\omega)$.

The Theorem follows now by the arguments given before Proposition 2.

Acknowledgements. I am indebted to Joel Lebowitz who first raised my interest to the type of questions discussed in this paper and to L. Gross who mentioned to me the work of Griffiths and Pearce. It is also a pleasure to thank M. Aizenman, J. Bricmont and S. Goldstein for discussions.

\section{References}

[Bil] Billingsley, P.: Probability and measure. Sec. Ed. New York: John Wiley 1986

[CCS] Chayes, J. T., Chayes, L., Schonmann, R. H.: Exponential decay of connectivities in the two dimensional Ising model. J. Stat. Phys. 49, 433-445 (1987)

[Com] Comets, F.: Grandes deviations pour des champs de Gibbs sur $Z^{d}$. C. R. Acad. Sci. Ser. I, 303, 511-513 (1986)

[FO] Föllmer, H., Orey, S.: Large deviations for the empirical field of a Gibbs measure. Preprint (1986), to appear in Ann. Probab.

[GP 1] Griffiths, R. B., Pearce, P. A.: Position-space renormalization-group transformations: Some proofs and some problems. Phys. Rev. Lett. 41, 917-920 (1978)

[GP 2] Griffiths, R. B., Pearce, P. A.: Mathematical properties of position-space renormalization-group transformations. J. Stat. Phys. 20, 499-545 (1979) 
[Gri] Griffiths, R. B.: Mathematical properties of renormalization-group transformations. Physica 106A, 59-69 (1981)

[Isr 1] Israel: R. B.: Existence of phase transitions for long range interactions. Commun. Math. Phys. 43, 59-68 (1975)

[Isr 2] Israel, R. B.: Convexity in the theory of lattice gases. Princeton, NJ: Princeton University Press 1979

[Isr 3] Israel, R. B.: Banach algebras and Kadanoff transformations in Random Fields, Vol. I, II (Esztergom, 1979), pp. 593-608. Collog. Math. Soc. Janos Bolyai, 27. Amsterdam: NorthHolland, 1981

[Koz] Kozlov, O. K.: Gibbs description of a system of random variables. Problemy Peredachi Informatsii 10, 94-103 (1974)

[Kun] Kunsch, H.: Non-reversible stationary measures for infinite interacting particle systems. Z. Wahrsch. Verw. Gebiete 66, 407-424 (1984)

[LS] Lebowitz, J. L., Schonmann, R. H.: Pseudo free energies and large deviations for non-Gibbsian FKG measures. Probab. Th. Rel. Fields 77, 49-64 (1988)

[O11] Olla, S.: Large deviations for Gibbs random fields. Preprint 1986, to appear in Probab. Th. Rel. Fields

[Rue] Ruelle, D.: Thermodynamic formalism. Reading, MA: Addison-Wesley 1978

[Rus] Russo, L.: The infinite cluster method in the two-dimensional Ising model. Commun. Math. Phys. 67, 251-266 (1979)

[Sch] Schonmann, R. H.: Second order large deviation estimates for ferromagnetic systems in the phase coexistence region, Commun. Math. Phys. 112,, 409-422 (1987)

[Sul] Sullivan, W. G.: Potentials for almost Markovian random fields. Commun. Math. Phys. 33, 61-74 (1973)

Communicated by M. Aizenman

Received March 9, 1988 
